\title{
Mapping LGBTQ+Youth Resource Density Across Four High HIV Prevalence Corridors in the US
}

\author{
Gregory Sallabank ${ }^{1}$ (1) - Tanaka M. D. Chavanduka ${ }^{1}$ - Alison R. Walsh ${ }^{1} \cdot$ Patrick Sullivan $^{2} \cdot$ James Wolfe $^{3}$. \\ Rebecca Filipowicz ${ }^{2}$ Erin E. Bonar ${ }^{4} \cdot$ Keith J. Horvath $^{5} \cdot$ Benyam Hailu $^{6} \cdot$ José Bauermeister $^{3} \cdot$ Rob Stephenson $^{1}$
}

Accepted: 14 October 2021 / Published online: 22 October 2021

(c) The Author(s), under exclusive licence to Springer Science+Business Media, LLC, part of Springer Nature 2021

\begin{abstract}
Introduction Studies using geospatial data to understand LGBTQ+-friendly sexual health and wellness resource availability have often focused on services catered to adults. While HIV rates have increased in adolescents in recent years, few studies have explored disparities in resource access for adolescent gay and bisexual men (AGBMSM).

Methods We used geospatial data of resources (collected and verified 2017-2018) from the iReach app to understand disparities in resource access for AGBMSM within and between 4 high HIV prevalence corridors in the US.

Results AGBMSM in non-metro areas had access to fewer resources and some rural counties had no LGBTQ+-friendly resources. Corridors comprising states with legacies of punitive laws targeting sexual and gender minorities demonstrate stark geographic disparities across the US.

Conclusions Policy-makers must understand the granularity of disparities within regions. Online resources may be able to surmount LGBTQ+ resource deserts. However, physical access to LGBTQ+-friendly services must be improved as a fundamental strategy for reducing HIV among AGBMSM.
\end{abstract}

Keywords Sexual health $\cdot$ HIV prevention $\cdot$ LGBTQ youth $\cdot$ Resource density $\cdot$ STI prevention $\cdot$ Adolescent health

\section{Introduction}

Adolescent and young adult gay, bisexual, and other men who have sex with men (AGBMSM; aged 13-24) are disproportionately affected by the HIV epidemic in the United

Gregory Sallabank

gsall@umich.edu

1 Center for Sexuality and Health Disparities, School of Nursing, University of Michigan, Ann Arbor, MI, USA

2 Rollins School of Public Health, Emory University, Atlanta, GA, USA

3 School of Nursing, University of Pennsylvania, Philadelphia, PA, USA

4 Dept of Psychiatry and Addiction Center, Injury Prevention Center, Center for Sexuality and Health Disparities, University of Michigan, Ann Arbor, MI, USA

5 Department of Psychology, San Diego State University, San Diego, CA, USA

6 Division of Clinical \& Health Services Research, National Institute On Minority Health and Health Disparities, National Institutes of Health, Bethesda, MD, USA
States (US). Between 2000 and 2010, the incidence of HIV infections among AGBMSM more than doubled (Johnson et al., 2014). In 2016, youth aged 13 to 24 years accounted for $21 \%$ of all new HIV diagnoses in the US, and $81 \%$ of these incident cases were among young gay and bisexual men (Centers for Disease Control \& Prevention, 2019). Yet, research examining geospatial resource locations and access and its impacts on HIV-related outcomes has generally focused on adult populations (Bauermeister et al., 2017, 2019; Pearce et al., 2006). Consequently, few programs or interventions have identified and verified LGBTQ+affirming local resources, including HIV prevention and screening resources that openly welcome AGBMSM (Bauermeister et al., 2018).

Structural vulnerability-including higher levels of unemployment, incarceration (Maiorana et al., 2019), and homelessness (Aidala et al., 2005) — has been associated with HIV risk in AGBMSM, particularly AGBMSM of color (Mackenzie, 2019; Tempalski et al., 2020). These structural factors are embedded in the sociopolitical landscape and often interact with individual experiences of racism and sexual minority-based stigma to increase HIV risk (Arrington-Sanders et al., 2020; Bourgois 
et al., 2017; Gamarel et al., 2018; Hatzenbuehler, 2017; Parker et al., 2017). In parallel, a lack of tailored, LGBTQ+-focused, or welcoming general health and support services can perpetuate sexuality-based stigma, creating additional barriers that can discourage AGBMSM from accessing important in-person services and resources (Fisher et al., 2018; McInroy et al., 2019; Pantelic et al., 2019). Thus, reduced access to appropriate services and support represents an important structural vulnerability that may intensify the cognitive and behavioral risk factors that, in turn, contribute to this population's disproportionate HIV burden (Arreola et al., 2015; Hill et al., 2020; Watson et al., 2020), increased STI risk (Poteat et al., 2019), and poor mental health outcomes that are endemic among AGBMSM (Eisenberg et al., 2019, 2020; Turpin et al., 2020).

These structural factors are malleable, and interventions aimed at reducing structural vulnerabilities and reducing disparities in relevant service availability may mediate negative health effects in AGBMSM (Matsick et al., 2020). Programs and interventions that create or expand health and well-being services tailored, or welcoming, to sexual minority youth can play a vital role in increasing AGBMSM's health (Coulter et al., 2019; Jadwin-Cakmak et al., 2020). Moreover, LGBTQ+ youth-directed and welcoming services can alleviate the disproportionate mental health burden felt by sexual minority adolescents (Lucassen et al., 2017; Walls et al., 2013). In the long term, increasing access to targeted services and interventions that foster self-efficacy and life skills during a critical period can increase positive health behaviors and coping mechanisms (Bauermeister et al., 2018), potentially reducing HIV burdens and disparities and improving physical and mental health outcomes across the life course in gay, bisexual, and other men who have sex with men (GBMSM) populations.

To date, there is limited research exploring regional variances in LGBTQ+ youth-appropriate and welcoming health and well-being resources (Martos et al., 2017). Found in an international sample, MSM never tested for HIV are more likely to be younger in age, have lower education status, and living outside a large urban area (Zimmermann et al., 2021). Some attention has been paid to disparities in access to LGBTQ+ health services between rural and urban communities, identifying a trend towards greater availability in urban areas (Martos et al., 2017). Analyses of HIV-focused and/or LGBTQ+ resources have been limited to smaller geographic areas: other small-scale spatial analyses have also found differential geographic access to LGBTQ+-appropriate services in Toronto neighborhoods (Fulcher \& Kaukinen, 2005), Miami-Dade County, Florida (Ganapati et al., 2010), and according to neighborhood socioeconomic status in Chicago (Mustanski et al., 2015; Rosentel et al., 2020). To date, little attention has focused upon comparing urban, rural, and intra- and inter-regional areas to improve our understanding of how location and differing sociopolitical contexts impact the health of AGBMSM. We are unaware of any previous studies that have compared LGBTQ+-inclusive health and wellness service access across, and within, large geographic areas in the US.

This ecological study aimed to describe levels of, and disparities in, the availability of LGBTQ+-inclusive and/ or affirming health and wellness resources in regions heavily impacted by the HIV/AIDS epidemic in the US. The overarching goal of this study was to assess and compare regional and county level per capita service availability in order to identify geographic areas with potentially underserved young LGBTQ+ populations. Identifying geographic disparities in access to services and programs fills a current gap in our broader understanding of how structural factors may contribute to the disproportionate HIV burden experienced by AGBMSM in the US, and the need for regionalspecific interventions to improve HIV testing and care outcomes.

\section{Methods}

Data for these analyses stem from a randomized control trial (RCT) which aims to test the efficacy of an e-delivered life skills intervention application (iReach) on cognitive and behavioral HIV-related outcomes for adolescent men (ages 13-18) who have same-sex attraction, in 4 geographically and racially diverse regions in the US heavily affected by HIV (Bauermeister et al., 2018). The iReach application includes information, goal-setting, peer-mentor sessions, and a searchable resource locator database of LGBTQ+ youth-friendly and inclusive community resources, across four health and well-being domains: sexual health and HIV; LGBTQ+ community; psychosocial (including intimate partner violence services); and other (e.g., food pantries, shelters). The resource locator is comprised of local services that have been verified as "LGBTQ+-affirming"-either designed for, or welcoming to, LGBTQ+ youth. The verification process adapted methodologies used in previous studies identifying HIV testing (Bauermeister et al., 2015) and PrEP providers (Siegler et al., 2017).

The intervention trial focused its sampling frame across four regions in the US: (1) Pacific-San Francisco, CA to San Diego, CA; (2) South-Atlantic-Washington, DC to Atlanta, GA; (3) East-North-Central-Chicago, IL to Detroit, MI; and (4) East-South-Central-Memphis, TN to New Orleans, LA. These regions were identified and selected by inspection of HIV prevalence rate maps on AIDSVu.org; eligible counties $(N=109)$ were those containing a major interstate highway connecting each region's two anchor cities (I-5: Pacific (San Francisco to San Diego); I-95: South-Atlantic (Washington, DC to Atlanta); I-94: East-North-Central (Chicago to Detroit); and I-55: East-South-Central (Memphis to 
New Orleans)). Each region includes urban, suburban, and rural counties, as classified by United States Department of Agriculture (USDA) (Parker, 2013).

\section{Resources and Service Providers}

The current study utilizes data from the iReach trial's database of LGBTQ+-inclusive service providers. As part of the iReach intervention, participants could access and search for local health and well-being service providers in the app-associated database of youth-friendly and LGBTQ+-inclusive public and private providers. The database contains eligible service providers in the 109 target counties; providers were identified and verified according to the trial's protocol (verification completed in 2018). Briefly, services/providers were identified using a list of Internet search terms for service types and location (county) and verified by study staff using provider website information, phone calls to confirm resource/service type and accessibility for LGBTQ+ youth, and follow-up emails when required. Providers were included in the iReach trial's database if they (1) were located in one of the 109 counties within the study's four recruitment corridors/regions; (2) provided youth-friendly or directed health or well-being services; and (3) confirmed that they were affirming of LGBTQ+ youth. Service providers were contacted directly by the study team in a two-part verification process including (1) a postcard with the choice to opt out and (2) a direct telephone call that asked to confirm that they welcomed and supported LGBTQ+ youth which also enabled study staff to gain broader insight of the organization. A service/resource provider's inclusiveness for both younger ages ( $<18$ years) and LGBTQ+/sexual and gender minorities was determined according to the process detailed in Drab et al. (2021). During the identification/verification process, staff noted in the trial's database each provider's offered services and categorized providers and their offered resources/services according to domain: (1) HIV/sexual health (HIV/SH) services: HIV testing, PrEP/PEP, HIV treatment/care, STI testing, other HIV service(s); (2) LGBTQ+ community resources: LGBTQ+ support group(s), LGBTQ+ youth-specific programs, other LGBTQ+ service(s); (3) psychosocial services: general support groups, substance use, mental health, IPV support, other psychosocial service(s); (4) other resources: food pantry, housing/shelter, school resources, or family support. Within the iReach WebApp, study participants could search for local resource/service providers according to domain (e.g., HIV services) or specific service (e.g., HIV testing). Providers who offered services spanning multiple domains were searchable according to all of their services and domains (for example, a provider who offered HIV testing and counselling could be accessed by searching for either HIV or Mental Health services).

\section{Additional Measures}

County-level sociodemographic characteristics were obtained from the US Census Bureau and American Community Survey 5-year (2013-2017) estimates: total and youth (ages 13-24) population size, population density, Hispanic/Latino and non-White populations, and population below the federal poverty line (United States Census Bureau, 2017). Analyzed counties were dichotomized by urbanicity based on their 2013 USDA ERS Rural-Urban Continuum Codes (Parker, 2013); "metro" counties included counties in metro areas (RUCC values 1-3) and "non-metro" counties included those with urban or completely rural populations (RUCC values: 4-9). County-level sociodemographic distributions, stratified by region and metro status, were assessed and compared using Kruskal-Wallis tests.

\section{Analysis}

For the current study, we used the iReach provider database to identify and categorize listed providers according to their offered services within the above four domains. Domains/services were not mutually exclusive, and providers could be categorized according to multiple domains/ services. We did not analyze each of the "other" domain services individually. For each provider within a given county/region, the number of domains covered and specific services offered was counted and aggregated to the county level. Regional differences in the county-average number of resource domain and service covered per provider were assessed with Kruskal-Wallis tests. We used a containerbased/county level per capita approach to create a proxy measure of county-level resource/service access, i.e., we normalized county-level provider counts by available county youth population data (aged 13-24) to arrive at a provider density per capita youth measure $(N$ providers per 10,000 youth population). Per capita measures have previously been used to estimate availability and access to healthcare resources and can be easily compared to benchmarks for county-level need (Andrilla et al., 2018; Davis et al., 2018; Konrad et al., 2009; US Department of Health and Human Services, Health Resources \& Service Administration, B. of H. W., 2021). Counties that did not contain any providers within a given domain or service were considered "resource deserts" for that domain or service. County-level provider density distributions were compared between study regions (and stratified by metro status) with Kruskal-Wallis tests. Correlations between county-level sociodemographic variables (Hispanic/Latino and non-White populations, and population below the federal poverty line) and provider densities were measured with Spearman's rank correlation coefficients (overall and stratified by study region) and 
interpreted according to Schober et al. (2018). Fisher transformations were used to determine correlations' statistical significance; correlations with statistically significant differences between regions (i.e., non-overlapping 95\% confidence intervals for 2 or more regions) are reported stratified by region. All statistical analyses were done in SAS 9.4 and $p$-values $<0.05$ were considered statistically significant. Service providers were geocoded from address data using the Geocode by Awesome Table tool. Regional maps illustrating provider densities were created using Mapbox, an opensource mapping platform, and ArcGIS Pro 2.6.3 (Esri Inc., 2020). County-level attributes were matched geometrically to the 2017 US Census shapefile using the five-digit Federal Information Processing Standard county code.

\section{Results}

Figure 1 maps the iReach study regions and Figs. 2, 3, and 4 show the county-level distribution of service providers and relative service availability (e.g., provider densities) across the study regions. Supplementary Figs. 1-4 present countylevel locator maps within each region. Table 1 presents mean county-level sociodemographic characteristics and provider densities (per 10,000 youth, ages 13-24) stratified by study region, across health and well-being domains and services. Table 2 presents the distributions of countylevel sociodemographics and resource/service densities in
East-South-Central and South-Atlantic regions, stratified by county metro status (the other regions did not contain any non-metro counties). Overall metro and non-metro meancounty resource and sociodemographic distributions are shown in Table 3. Spearman's rank correlation coefficients for sociodemographic variables and service provider densities are shown in Table 4 . The total numbers of providers and providers offering each analyzed service domain/service in each region are presented in Supplementary Table 1, and Supplementary Tables 3 and 4 present the number of counties without any local providers offering specific services, within each region and across metro/non-metro counties.

\section{Sociodemographics}

Study regions had significantly different county-level sociodemographic characteristics, in terms of population, population density, and age, ethnic/racial, and socioeconomic compositions. Pacific region counties $(N=14)$ had, on average, higher total, minority populations and population density than other regions, whereas East-South-Central region counties $(N=27)$ had lower populations, population densities, Hispanic/Latino populations, and higher poverty levels than the other three regions. Although South-Atlantic region counties $(N=57)$ had higher minority populations than those in the East-North-Central region $(N=11)$, the two regions were otherwise demographically similar. In terms of urbanicity, $100 \%$ of the Pacific- and East-North-Central

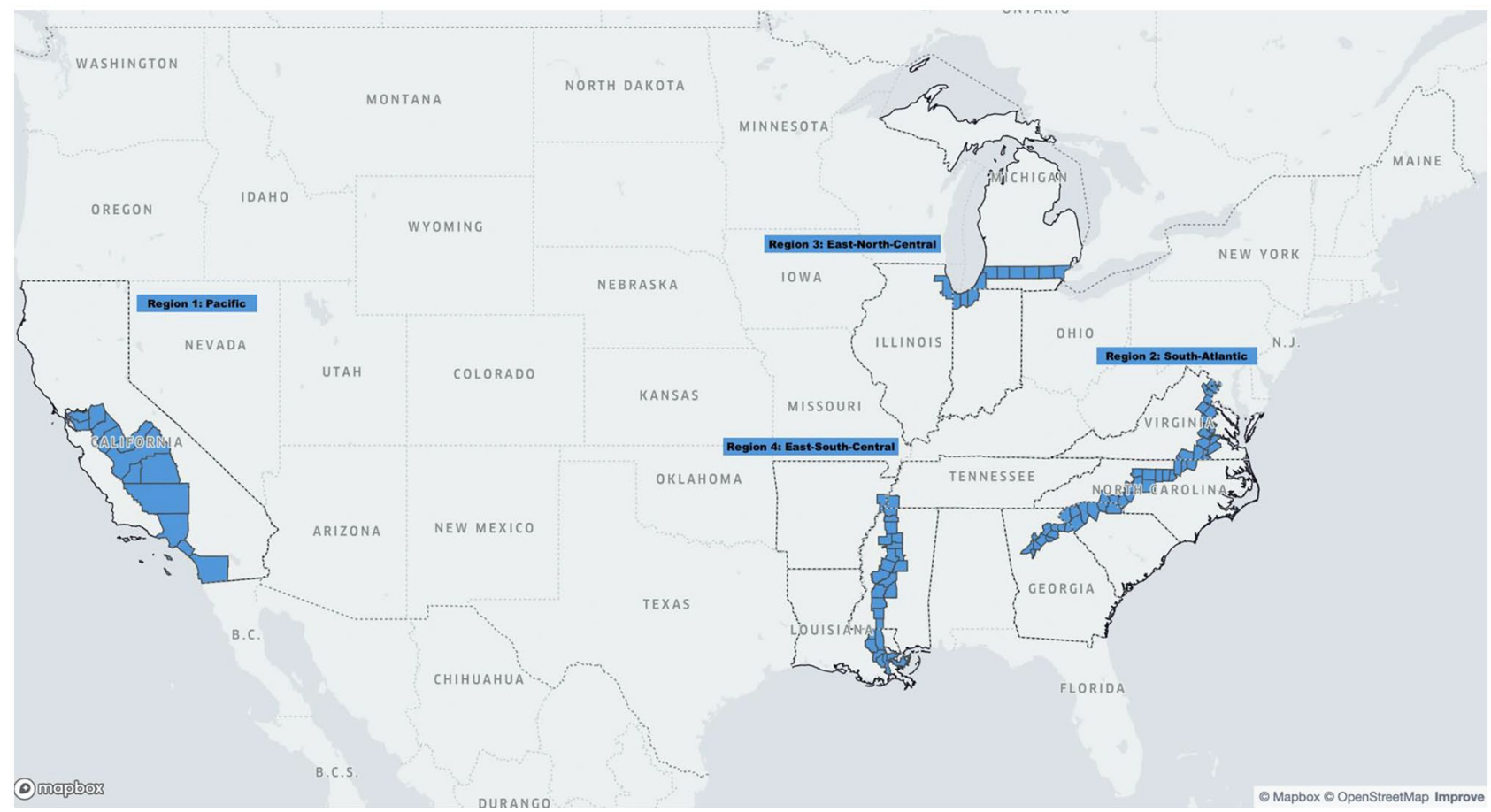

Fig. 1 Map of 4 US regions used in the iReach study and included counties, US, 2015 

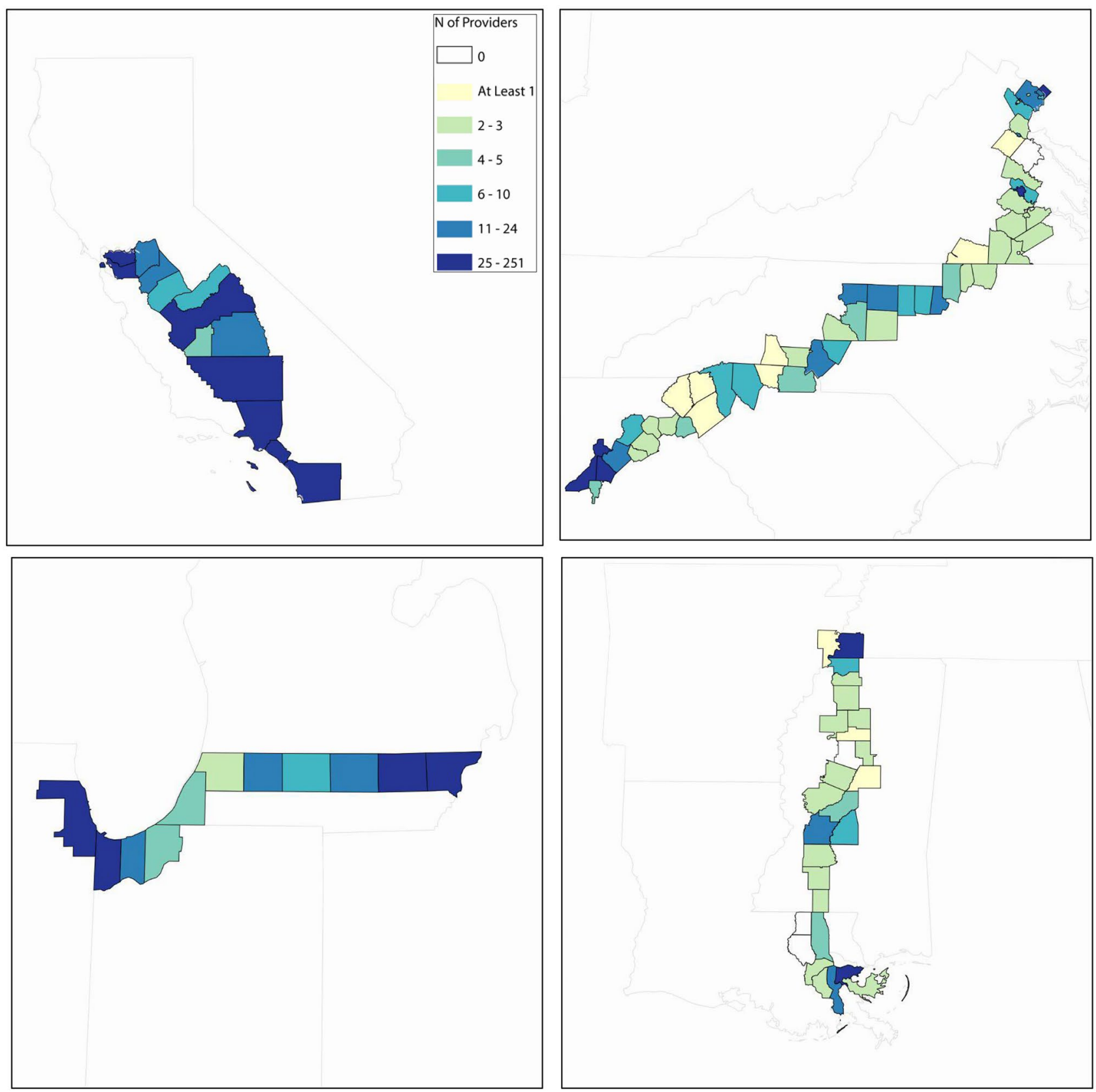

Fig. 2 Number of providers per county

regions, $75 \%$ of South-Atlantic region $(N=43)$, and $63 \%$ of East-South-Central region $(N=17)$ counties were classified as metro. The 85 metro counties were significantly more populated, diverse, and affluent than the 24 non-metro counties. Most metro status differences persisted when stratified by region. In both South-Atlantic and East-South-Central regions, metro counties were significantly more densely populated, racially/ethnically diverse, and affluent than non-metro counties (\% poverty difference not significant in East-South-Central region). Notably, East-South-Central region's non-metro counties had higher mean poverty and lower Hispanic/Latino populations than counties in the other regions, regardless of metro status.

\section{Service Providers}

A total of 1793 unique service providers in 109 counties were listed in the baseline iReach database (as of 2018; see Fig. 2$), 45 \%(N=797)$ of which were in the Pacific region, $29 \%(N=513)$ in the South-Atlantic region, $18 \%(N=324)$ in the East-North-Central region, and 9\% $(N=159)$ in the EastSouth-Central region; 6 counties ( 3 in the South-Atlantic 

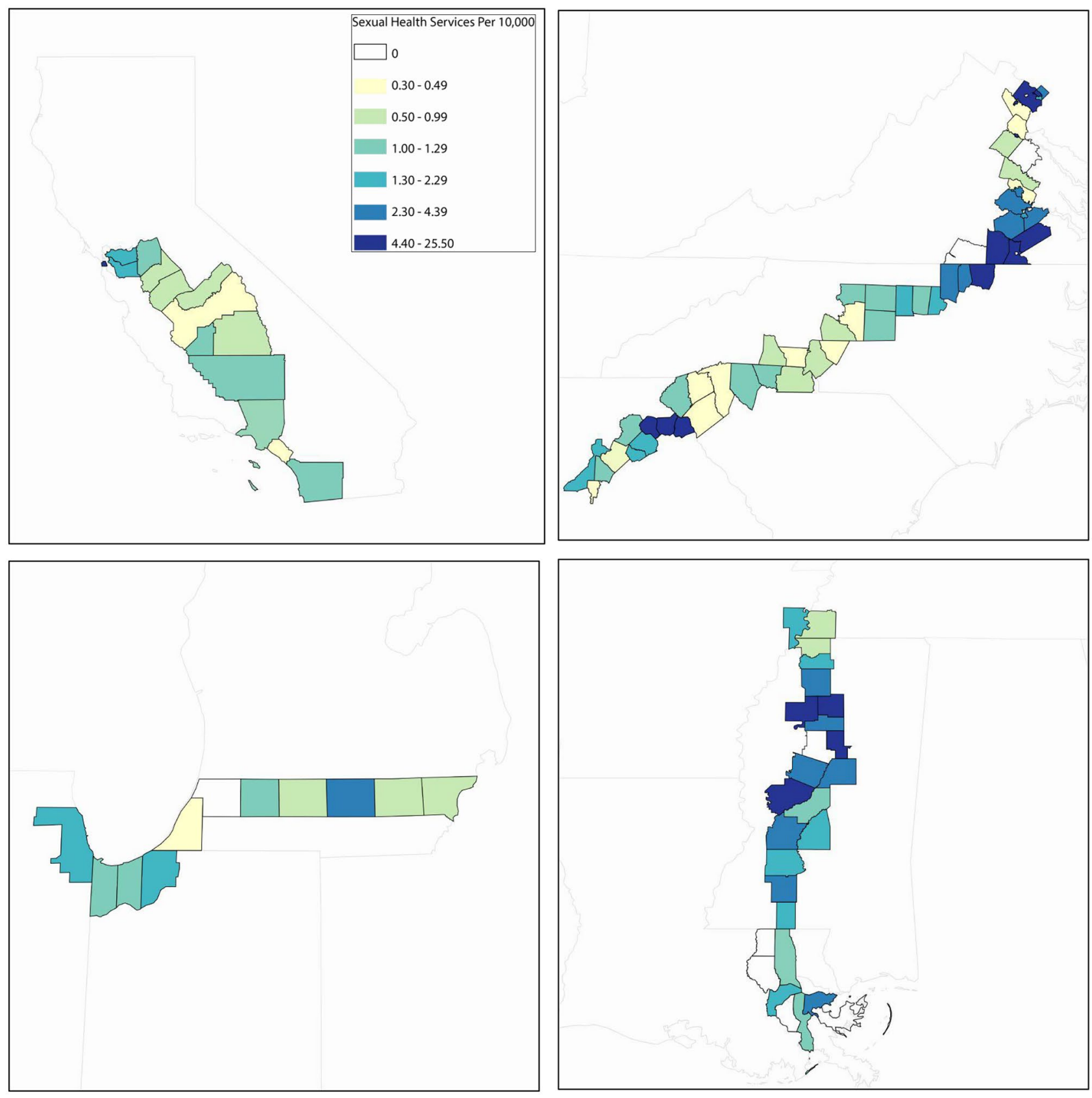

Fig. $3 \mathrm{HIV} / \mathrm{sexual}$ health service providers ( $N$ per 10,000 youth)

region, 3 in the East-South-Central region) did not have any relevant providers. Collectively, service providers offered an average of $2.68 \pm 1.58$ of the enumerated services, across $1.55 \pm 0.76$ domains; providers in the East-North-Central region were the most comprehensive and providers in the East-South-Central region, the least, in terms of both available services and covered resource domains $(p<0.05)$. Counties' percent of racial/ethnic minority residents and percent of persons living below the federal poverty line had weak to moderate positive monotonic relationships with overall provider density $(p<0.05)$; there were no significant differences in these correlations between study regions.

\section{HIV/Sexual Health Domain Services}

Across study regions, $42-48 \%$ of identified service providers offered at least one HIV/SH service (see Fig. 3). In the South-Atlantic, East-North-Central, and East-SouthCentral regions, there were $3(5 \%), 1(9 \%)$, and $5(19 \%)$ counties without any youth-HIV/SH providers, respectively. 

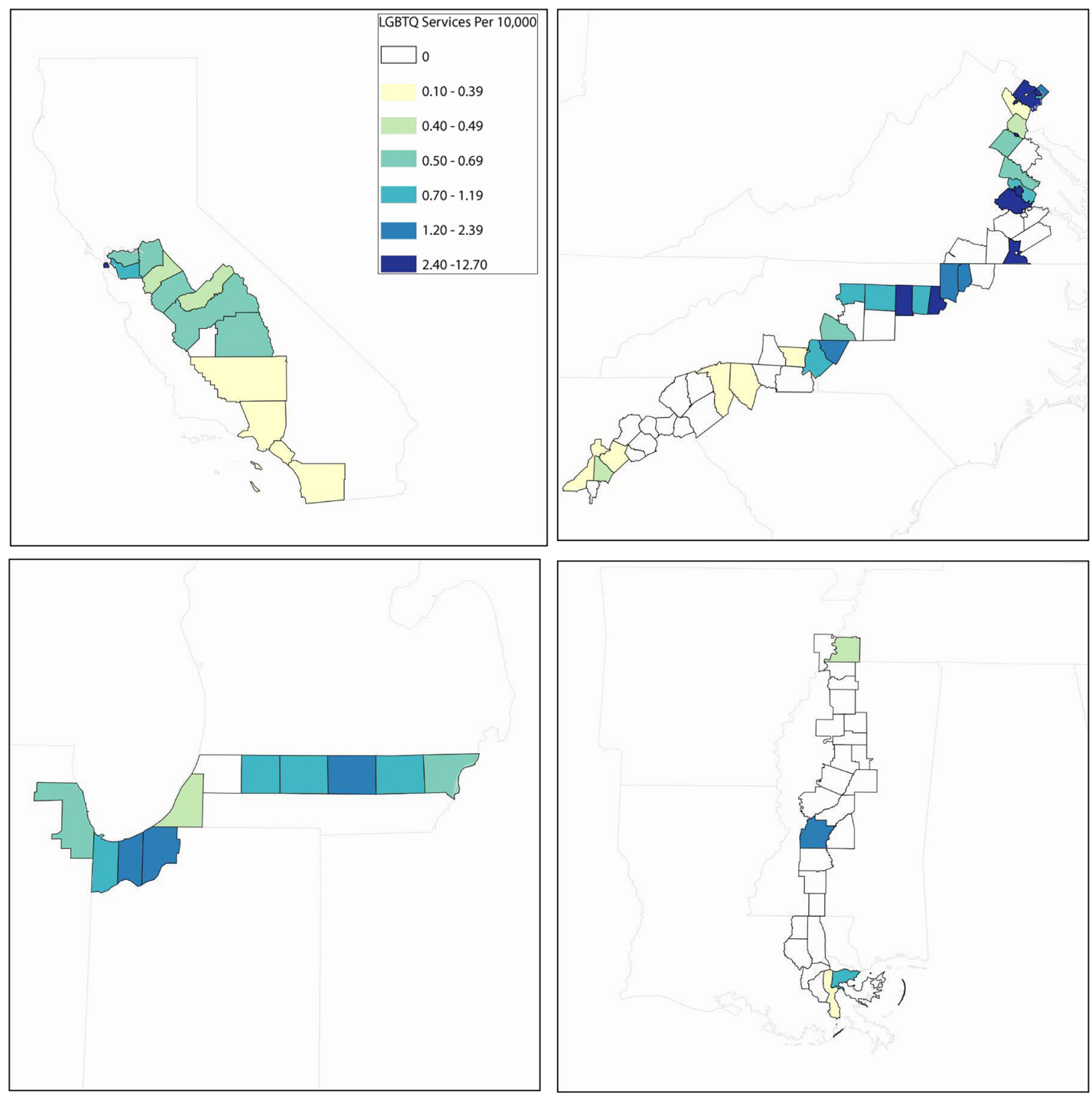

Fig. 4 LGBTQ+ community service provider density ( $N$ per 10,000 youth)

The mean county-level HIV/SH provider density was highest in the East-South-Central region $(2.59 \pm 2.99$ per 10,000 youth) and lowest in the East-North-Central region $(1.04 \pm 0.77$ per 10,000 youth), although there were no significant differences in densities between study regions $(p>0.05)$. Provider densities for this domain exhibited statistically significant monotonic associations with countylevel sociodemographics. Across all study regions, Hispanic/Latino populations were negatively correlated with $\mathrm{HIV/sexual} \mathrm{health} \mathrm{provider} \mathrm{density} \mathrm{whereas} \mathrm{total} \mathrm{non-White}$ population percentages were positively correlated with access in this domain. The correlations between counties' populations living in poverty and HIV/sexual health providers were differential across study regions and were statistically significant in the South-Atlantic region and the EastNorth-Central region. South-Atlantic counties had a weak to moderate positive monotonic relationship between poverty and sexual health providers whereas East-North-Central counties' densities were negligibly to strongly negatively correlated with poverty. 
Table 1 Mean county-level sociodemographics and sexual minority youth-inclusive service provider densities $(N$ providers per 10,000 youth (ages 13-24) population), stratified by iREACH study region,
US, 2015. Data are $N$ or mean standard \pm deviation (SD). Differences in regional county-means were assessed with Kruskal-Wallis tests

\begin{tabular}{|c|c|c|c|c|c|}
\hline & Pacific & South-Atlantic & East-North-Central & East-South-Central & $p$-value \\
\hline \multicolumn{6}{|l|}{ Sociodemographic characteristics } \\
\hline Counties $(N)$ & 14 & 57 & 11 & 27 & \\
\hline Population $(\times 10,000)$ & $174.68 \pm 261.35$ & $22.43 \pm 27.89$ & $80.59 \pm 153.44$ & $11.87 \pm 19.74$ & $<0.0001^{* * * *}$ \\
\hline Population density $\left(N /\right.$ land $\left.\mathrm{mi}^{2}\right)$ & $881.81 \pm 2099.95$ & $621.72 \pm 1076.51$ & $463.01 \pm 713.59$ & $121.46 \pm 248.80$ & $<0.0001^{\text {**** }}$ \\
\hline Youth population (\% of population; ages $13-24$ ) & $16.68 \pm 2.31$ & $15.68 \pm 2.54$ & $16.93 \pm 3.25$ & $15.90 \pm 1.52$ & $0.04^{*}$ \\
\hline Hispanic/Latino (\% of population) & $42.97 \pm 14.75$ & $9.11 \pm 6.29$ & $9.04 \pm 6.87$ & $3.76 \pm 3.04$ & $<0.0001^{* * * *}$ \\
\hline Non-White (\% of population) & $64.12 \pm 6.42$ & $40.54 \pm 7.53$ & $29.15 \pm 14.76$ & $48.55 \pm 15.99$ & $<0.0001^{* * * *}$ \\
\hline Below federal poverty line ( $\%$ of population) & $16.37 \pm 5.55$ & $14.63 \pm 5.68$ & $15.71 \pm 3.53$ & $22.05 \pm 7.85$ & $<0.0001^{* * * *}$ \\
\hline \multicolumn{6}{|l|}{ Service provider densities ( $N$ per 10,000 youth) } \\
\hline \multicolumn{6}{|l|}{ Resource domain Service offered } \\
\hline All providers & $2.67 \pm 2.49$ & $4.51 \pm 6.23$ & $3.16 \pm 1.25$ & $4.24 \pm 3.25$ & 0.17 \\
\hline HIV/sexual health & $1.20 \pm 1.16$ & $2.29 \pm 2.87$ & $1.04 \pm 0.77$ & $2.59 \pm 2.99$ & 0.35 \\
\hline HIV testing & $0.86 \pm 0.81$ & $2.03 \pm 2.47$ & $0.90 \pm 0.70$ & $2.51 \pm 3.00$ & 0.12 \\
\hline PrEP/PEP & $0.18 \pm 0.19$ & $0.50 \pm 1.34$ & $0.23 \pm 0.26$ & $0.15 \pm 0.38$ & $0.02^{*}$ \\
\hline HIV treatment or care & $-{ }^{\mathrm{a}}$ & $1.24 \pm 2.07$ & $0.25 \pm 0.30$ & $0.16 \pm 0.37$ & $<0.0001^{* * * *}$ \\
\hline STI testing & $0.73 \pm 0.74$ & $1.74 \pm 2.48$ & $0.48 \pm 0.46$ & $2.35 \pm 3.03$ & 0.06 \\
\hline Other HIV service(s) & $0.30 \pm 0.43$ & $0.23 \pm 1.00$ & $0.13 \pm 0.20$ & $0.03 \pm 0.14$ & $<0.0001^{\text {**** }}$ \\
\hline LGBTQ+ community & $0.67 \pm 1.06$ & $0.70 \pm 1.20$ & $0.87 \pm 0.53$ & $0.11 \pm 0.30$ & $<0.0001^{\text {**** }}$ \\
\hline LGBTQ+ support group(s) & $0.30 \pm 0.46$ & $0.44 \pm 1.00$ & $0.20 \pm 0.19$ & $0.06 \pm 0.18$ & $<0.001^{* * * *}$ \\
\hline Programming for LGBTQ+ youth & $0.35 \pm 0.70$ & $0.22 \pm 0.43$ & $0.29 \pm 0.26$ & $0.05 \pm 0.19$ & $<0.001^{* * * *}$ \\
\hline Other service(s) & $0.21 \pm 0.31$ & $0.19 \pm 0.37$ & $0.47 \pm 0.47$ & $0.01 \pm 0.03$ & $<0.0001^{* * * *}$ \\
\hline Psychosocial & $1.83 \pm 1.88$ & $2.68 \pm 5.48$ & $2.61 \pm 1.19$ & $1.92 \pm 1.65$ & $0.03^{*}$ \\
\hline Support group(s) & $0.69 \pm 0.67$ & $0.83 \pm 2.39$ & $1.26 \pm 0.87$ & $0.79 \pm 1.02$ & $0.02^{*}$ \\
\hline Substance use support & $0.47 \pm 0.53$ & $0.90 \pm 1.83$ & $1.21 \pm 0.72$ & $0.83 \pm 1.10$ & $0.02^{*}$ \\
\hline Mental health support & $1.27 \pm 1.17$ & $1.62 \pm 3.82$ & $1.90 \pm 1.08$ & $1.78 \pm 1.69$ & $<0.001^{* * * *}$ \\
\hline IPV support & $0.38 \pm 0.22$ & $0.16 \pm 0.43$ & $0.23 \pm 0.25$ & $0.13 \pm 0.35$ & $<0.001^{* * * *}$ \\
\hline Other service(s) & $0.20 \pm 0.43$ & $0.61 \pm 1.20$ & $0.28 \pm 0.41$ & $0.08 \pm 0.25$ & $<0.01^{* *}$ \\
\hline Other $^{b}$ & $0.51 \pm 0.37$ & $0.49 \pm 1.08$ & $0.39 \pm 0.37$ & $0.16 \pm 0.33$ & $<0.01^{* * *}$ \\
\hline
\end{tabular}

${ }^{*} p<0.05 ;{ }^{* *} p<0.01 ;{ }^{* * *} p<0.001$

${ }^{a}$ Data unavailable (not recorded in study database)

${ }^{\mathrm{b}}$ Includes food pantries, housing/shelter, school resources/programming, family support resources

Overall, metro counties had significantly lower HIV/ $\mathrm{SH}$ provider and service densities than non-metro counties, and this trend was also seen when the South-Atlantic and East-South-Central regions were stratified by metro status. Although overall densities of HIV/SH service providers, and HIV and STI testing provider densities, did not significantly differ by study region $(p>0.05)$, there were significant differences in PrEP/PEP, HIV treatment/ care, and other HIV service provider densities between regions $(p<0.05)$. Within the South-Atlantic and EastSouth-Central regions, HIV/SH service providers were skewed in favor of metro counties. In the South-Atlantic region, $79 \%$ of non-metro and $65 \%$ of metro counties did not have any PrEP/PEP providers, and in the East-SouthCentral region, $100 \%$ of non-metro counties did not house any PrEP/PEP, HIV treatment/care, or other HIV service providers.

\section{LGBTQ+Community Domain Services}

Across study regions, $12-30 \%$ of identified youth-friendly service providers offered at least one service or program designed or targeted at LGBTQ+ youth (see Fig. 4) in addition to broadly welcoming LGBTQ+ youth across their service domains. Mean county-level LGBTQ+ community provider density was highest in the East-North-Central region and lowest in the East-South-Central region $(p<0.0001)$. Additionally, the provider distributions were particularly skewed across counties in the East-South-Central region, where $85 \%$ of counties did not have any LGBTQ+ community service providers. The East-South-Central region counties also had the lowest levels of access to services within the LGBTQ+ community domain, including support groups, programming, and other services $(p<0.001$ for all). Non-metro counties had significantly lower LGBTQ+ provider and service densities 
Table 2 Mean county-level sociodemographics and sexual minority youth-inclusive service provider densities ( $N$ providers per 10,000 youth (ages 13-24) population) by county metro status in iREACH's South-Atlantic (SA) and East-South-Central (ESC) study regions,
2015. The Pacific and East-North-Central regions did not have any non-metro counties and were thus not included in this analysis. Data are $N$ or mean standard deviation $\pm(\mathrm{SD})$. Differences in countymeans by metro status were assessed with Kruskal-Wallis tests

\begin{tabular}{|c|c|c|c|c|c|c|}
\hline & \multicolumn{3}{|c|}{ South-Atlantic (SA) } & \multicolumn{3}{|c|}{ East-South-Central (ESC) } \\
\hline & Metro & Non-metro & $P$-value & Metro & Non-metro & $P$-value \\
\hline \multicolumn{7}{|l|}{ Sociodemographics } \\
\hline Counties $(N)$ & 43 & 14 & & 17 & 10 & \\
\hline Population $(\times 10,000)$ & $27.95 \pm 30.01$ & $5.47 \pm 5.49$ & $<0.001^{* * *}$ & $17.60 \pm 23.21$ & $2.14 \pm 1.09$ & $<0.001^{* * *}$ \\
\hline Population density $\left(\mathrm{N} / \mathrm{land} \mathrm{mi}^{2}\right)$ & $771.4 \pm 1181.20$ & $161.97 \pm 427.36$ & $<0.0001^{* * *}$ & $182.22 \pm 299.88$ & $18.18 \pm 10.99$ & $<0.01^{* * *}$ \\
\hline Youth population (\%; ages 13-24) & $15.98 \pm 2.75$ & $14.74 \pm 1.41$ & 0.09 & $16.08 \pm 1.38$ & $15.60 \pm 1.78$ & 0.37 \\
\hline Hispanic/Latino (\%) & $10.53 \pm 6.57$ & $4.75 \pm 1.95$ & $<0.001^{* * * k}$ & $4.83 \pm 3.22$ & $1.93 \pm 1.55$ & $<0.01^{* *}$ \\
\hline Non-White (\%) & $41.55 \pm 16.94$ & $37.43 \pm 19.55$ & 0.42 & $47.38 \pm 16.99$ & $50.52 \pm 14.78$ & 0.81 \\
\hline Below federal poverty line (\%) & $12.96 \pm 4.96$ & $19.77 \pm 4.7$ & $<0.001^{\text {**** }}$ & $19.53 \pm 6.84$ & $26.33 \pm 7.92$ & 0.06 \\
\hline \multicolumn{7}{|c|}{ Service provider densities ( $N$ per 10,000 youth) } \\
\hline \multicolumn{7}{|l|}{ Resource domain Service offered } \\
\hline All providers & $3.84 \pm 6.33$ & $6.55 \pm 5.62$ & $0.02^{*}$ & $3.20 \pm 2.12$ & $6 \pm 4.14$ & $<0.05^{*}$ \\
\hline HIV/sexual health & $1.52 \pm 2.14$ & $4.63 \pm 3.59$ & $<0.001^{* * * *}$ & $1.40 \pm 1.32$ & $4.63 \pm 3.92$ & $<0.01^{* *}$ \\
\hline HIV testing & $1.47 \pm 2.14$ & $3.75 \pm 2.67$ & $<0.001^{\text {*a*k }}$ & $1.27 \pm 1.22$ & $4.63 \pm 3.92$ & $<0.01^{* *}$ \\
\hline $\mathrm{PrEP} / \mathrm{PEP}$ & $0.39 \pm 1.07$ & $0.84 \pm 1.95$ & 0.61 & $0.24 \pm 0.46$ & 0 & 0.06 \\
\hline HIV treatment or care & $1.19 \pm 2.15$ & $1.41 \pm 1.87$ & 0.84 & $0.26 \pm 0.45$ & 0 & $0.03^{*}$ \\
\hline STI testing & $1.18 \pm 2.08$ & $3.47 \pm 2.89$ & $<0.001^{\text {*冰* }}$ & $1.12 \pm 1.22$ & $4.45 \pm 4.02$ & $<0.01^{* *}$ \\
\hline Other HIV service(s) & $0.03 \pm 0.08$ & $0.87 \pm 1.93$ & 0.36 & $0.05 \pm 0.18$ & 0 & 0.27 \\
\hline LGBTQ+ community & $0.65 \pm 0.93$ & $0.85 \pm 1.84$ & 0.19 & $0.17 \pm 0.37$ & 0 & 0.10 \\
\hline LGBTQ+ support group(s) & $0.37 \pm 0.61$ & $0.64 \pm 1.76$ & 0.17 & $0.10 \pm 0.23$ & 0 & 0.10 \\
\hline Programming for LGBTQ+ youth & $0.25 \pm 0.45$ & $0.15 \pm 0.39$ & 0.14 & $0.09 \pm 0.23$ & 0 & 0.17 \\
\hline Other service(s) & $0.19 \pm 0.33$ & $0.19 \pm 0.49$ & 0.15 & $0.01 \pm 0.04$ & 0 & 0.44 \\
\hline Psychosocial & $2.65 \pm 5.90$ & $2.76 \pm 4.09$ & 0.56 & $1.86 \pm 1.25$ & $2.01 \pm 2.26$ & 0.80 \\
\hline Support group(s) & $0.92 \pm 2.68$ & $0.55 \pm 1.1$ & 0.15 & $0.88 \pm 1.02$ & $0.63 \pm 1.06$ & 0.34 \\
\hline Substance use support & $0.83 \pm 1.76$ & $1.10 \pm 2.08$ & 0.25 & $0.96 \pm 1.13$ & $0.63 \pm 1.06$ & 0.26 \\
\hline Mental health support & $1.61 \pm 4.20$ & $1.64 \pm 2.43$ & 0.31 & $1.64 \pm 1.32$ & $2.01 \pm 2.26$ & 1.00 \\
\hline IPV support & $0.11 \pm 0.21$ & $0.32 \pm 0.79$ & 0.56 & $0.20 \pm 0.43$ & 0 & $0.04^{*}$ \\
\hline Other service(s) & $0.54 \pm 0.95$ & $0.83 \pm 1.78$ & 0.54 & $0.13 \pm 0.31$ & 0 & 0.10 \\
\hline Other $^{\mathrm{a}}$ & $0.43 \pm 0.74$ & $0.69 \pm 1.79$ & 0.22 & $0.25 \pm 0.39$ & 0 & $0.04^{*}$ \\
\hline
\end{tabular}

${ }^{*} p<0.05 ;{ }^{* *} p<0.01 ;{ }^{* * *} p<0.001$

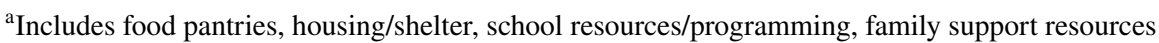

than non-metro counties; in the East-South-Central region, non-metro counties had no LGBTQ+ community providers, and in the South-Atlantic region, $71 \%$ of non-metro counties had no LGBTQ+ community providers. LGBTQ+community provider densities exhibited statistically significant, weak to moderate positive correlations with counties' Hispanic/Latino population shares in the South-Atlantic region. Overall, poverty was negatively correlated with LGBTQ+ community provider density (negligible to weakly negative).

\section{Psychosocial Domain Services}

Between 52 and $77 \%$ of identified service providers offered at least one youth-friendly psychosocial service or program. Mean county-level psychosocial provider density was highest in the South-Atlantic and East-North-Central regions and lowest in Pacific and East-South-Central regions $(p=0.03)$. Service and provider access in this domain was somewhat more evenly distributed than other domains; all counties in the Pacific region and East-North-Central region had at least 1 psychosocial provider, and only $19 \%$ and $22 \%$ of counties in South-Atlantic and East-South-Central regions, respectively, were lacking psychosocial providers, respectively. Within the South-Atlantic and East-SouthCentral regions, metro counties had lower psychosocial provider densities than non-metro counties, although the differences were not significant. Within this domain, counties had greater access to general mental health support services than other psychosocial services, particularly IPV support, which had the lowest provider densities across all regions. Psychosocial resource availability was not significantly correlated with the analyzed sociodemographic variables. 





Table 4 Spearman's rank correlation coefficients and 95\% confidence intervals (CIs) for bivariate correlations between county-level sociodemographic variables and sexual and gender minority youth-inclusive service provider densities ( $N$ providers per 10,000 youth (ages 13-24) population), US, 2015. For each sociodemographic character- istic and service provider domain, correlations for the 4 study regions were run collectively and individually. Regionally stratified correlations are presented if there were statistically significant differences between regions (i.e., non-overlapping 95\% CIs for at least 2 regions)

\begin{tabular}{|c|c|c|c|c|c|}
\hline \multirow[b]{2}{*}{$\begin{array}{l}\text { Sociodemographic } \\
\text { characteristic \& service } \\
\text { provider densities }(N \\
\text { per } 10,000 \text { youth) }\end{array}$} & \multirow[b]{2}{*}{ All counties $(N=109)$} & \multicolumn{4}{|c|}{ Regionally stratified results } \\
\hline & & Pacific $(N=14)$ & $\begin{array}{l}\text { South-Atlantic } \\
(N=57)\end{array}$ & $\begin{array}{l}\text { East-North-Central } \\
(N=11)\end{array}$ & $\begin{array}{l}\text { East-South-Central } \\
(N=27)\end{array}$ \\
\hline \multicolumn{6}{|l|}{$\begin{array}{l}\text { Hispanic/Latino (\% of } \\
\text { population) }\end{array}$} \\
\hline Total providers & $-0.17(-0.35,0.02)$ & & & & \\
\hline HIV/sexual health & $-0.23(-0.40,-0.05)^{*}$ & & & & \\
\hline $\begin{array}{l}\text { LGBTQ+ commu- } \\
\text { nity }\end{array}$ & & $-0.16(-0.64,0.41)$ & $0.46(0.23,0.64)^{* *}$ & $-0.44(-0.82,0.22)$ & $0.27(-0.12,0.59)$ \\
\hline Psychosocial & $0.05(-0.14,0.23)$ & & & & \\
\hline Other $^{\mathrm{a}}$ & $0.45(0.28,0.58)^{* * *}$ & & & & \\
\hline \multicolumn{6}{|l|}{$\begin{array}{l}\text { Non-White (\% of } \\
\text { population }\end{array}$} \\
\hline Total providers & $0.20(0.01,0.37)^{*}$ & & & & \\
\hline HIV/sexual health & $0.23(0.04,0.40)^{*}$ & & & & \\
\hline $\begin{array}{l}\text { LGBTQ+ commu- } \\
\text { nity }\end{array}$ & $0.18(-0.01,0.35)$ & & & & \\
\hline Psychosocial & $0.14(-0.05,0.32)$ & & & & \\
\hline Other $^{\mathrm{a}}$ & $0.28(0.09,0.44)^{* *}$ & & & & \\
\hline \multicolumn{6}{|l|}{$\begin{array}{l}\text { Below federal poverty } \\
\text { line ( } \% \text { of popula- } \\
\text { tion) }\end{array}$} \\
\hline Total providers & $0.27(0.09,0.44)^{* *}$ & & & & \\
\hline HIV/sexual health & & $-0.51(-0.82,0.02)$ & $0.38(0.13,0.58)^{* *}$ & $-0.61(-0.89,-0.02)^{*}$ & $0.47(0.11,0.72)$ \\
\hline $\begin{array}{l}\text { LGBTQ+ commu- } \\
\text { nity }\end{array}$ & $-0.21(-0.38,-0.02)^{* *}$ & & & & \\
\hline Psychosocial & $0.05(-0.14,0.24)$ & & & & \\
\hline Other $^{\mathrm{a}}$ & $-0.10(-0.28,0.09)$ & & & & \\
\hline
\end{tabular}

${ }^{*} p<0.05 ;{ }^{* *} p<0.01 ;{ }^{* * *} p<0.001$

${ }^{\mathrm{a}}$ Includes food pantries, housing/shelter, school resources/programming, family support resources

\section{Other Services}

Across the Pacific, South-Atlantic, and East-North-Central regions, food pantries, housing/shelter, school resources and programs, and family support resources had lower average county-level availability than other health and wellness domains. In the South-Atlantic region, non-metro counties had higher "other" service provider densities than metro counties, although the distributions were not statistically significantly different. In the East-South-Central region, the relationship between metro status and "other" service density was reversed and these services were concentrated in 6 of the 17 metro counties in this region $(p=0.04)$. Other services were weak to moderately positively correlated with county race/ethnicity ( $p<0.01$ for Hispanic/Latino population and non-White population).

\section{Discussion}

Our analysis represents a first step in describing and addressing the availability and disparities in LGBTQ+ youth-directed resources in the US. We identified disparities in county-level distributions of sexual and gender minority-welcoming health and wellness resource providers across four distinct corridors heavily impacted by the HIV/AIDS epidemic in the US. While generating a starting point for future in-depth geospatial analyses of youth-directed health and wellness resource availability, 
we found noteworthy disparities in local access to relevant service providers and resources across a wide range of service domains, both between and within the study regions. Moreover, disparities in resource and provider availability were also found between metro and non-metro counties. Given the generally weak to moderate correlations between poverty and provider availability this study identified, these disparities are likely not purely the result of differing monetary resources. Within South-Atlantic and East-South-Central counties, providers and resources were concentrated in metro areas; multiple non-metro counties in these areas were "resource deserts," in that they did not have any LGBTQ+ youth-inclusive providers.

The stark differences in numbers of LGBTQ+ community providers between US regions highlight the geographic disparities in the availability of programs that can play important roles in reducing sexual minority stigma (both external and internalized) and improving outcomes in AGBMSM (Matsick et al., 2020). In the South-Atlantic region, $46 \%$ of analyzed counties did not have any identified LGBTQ+ community providers, and in the South-Central region, $85 \%$ of counties were similarly lacking. Although legal rights and protections for sexual minorities have generally improved in the US in recent years (Movement Advancement Project, 2020a), some of the resource deserts we identified are located in states with legacies of punitive laws targeting sexual and gender minorities, as well as lower levels of legal protections and non-discrimination statutes (e.g., South Carolina, Mississippi) (Movement Advancement Project, 2020b). In comparison, the other two regions each had only one county without any LGBTQ+ community providers. Additionally, there were no LGBTQ+ community providers in any of the non-metro counties in the EastSouth-Central region and in $71 \%$ of non-metro counties in the South-Atlantic corridor. This result indicates the need to address structural-level access and availability of inclusive health and wellness resources for LGBTQ+ youth in many areas within the US. Furthermore, the identified regional and county-level disparities support the need to develop and fund telemedicine and virtual online resources, such as the iReach intervention, for LGBTQ+ youth, as large numbers of vulnerable sexual minority youth appear to lack local access to these important resources.

Contrary to the assumed equitable distribution of resource access in the US based on need, our results suggest that container-based/per capita density estimates of access may not accurately represent true geographical access to services for this population. Within regions, metro counties were both better resourced and had lower poverty rates, on average, than their non-metro counterparts. Although counties in the EastSouth-Central region had the highest average per capita HIV/ SH provider densities, $19 \%$ of counties in this region did not have any HIV/SH service providers, resulting in a large portion of this region's vulnerable youth lacking local access to important resources. This finding is particularly significant given the region's high HIV prevalence- $52 \%$ of new HIV diagnoses in 2017 in the US were in the South (Centers for Disease Control \& Prevention, 2019). Notably, HIV/SH continuum of care services (HIV treatment/care) and HIV prevention medication providers (PEP/PrEP) were absent from non-metro counties in the East-South-Central region. This result highlights the granularity of disparities in access within regions in the US; that is, local, county, or community-level service and resource availability does not necessarily follow larger regional trends. Moreover, the South continues to have a diagnosis rate 50\% higher (65\% higher in the Deep South) than that of any other region. Diagnoses in the Deep South increased among some groups, including the MSM. Our findings underscore the need to explore and characterize the relationships between geospatial factors and HIV risk behaviors in highly populated urban areas (Bauermeister et al., 2017; Fulcher \& Kaukinen, 2005; Ganapati et al., 2010) and rural and non-metro areas alike (Farmer et al., 2016), which reinforces the understanding that levels of utilization of HIV prevention services are lower for MSM in rural areas (McKenney et al., 2018; Sullivan et al., 2020). Resource allocation based on HIV incidence, prevalence, and risk should consider geospatial characteristics and urbanicity. Based on our findings, however, it is imperative that policy-makers avoid larger-scale comparisons of resource density (e.g., regional, states) as they may result in ecological fallacies that obscure disparities in service access and availability at the local or community level.

Neighborhoods and locales may also be segregated according to sexual orientation/identity where there is a high GBMSM population. Although these areas may have greater HIV prevention resources, individuals may be more susceptible to HIV transmission due to higher HIV prevalence among partners when compared to other areas (Kelley et al., 2012; Latkin et al., 2013). In southern communities where certain sexual identities and/or practices may be more stigmatized (Hill et al., 2020), youth are more likely to experience geographically segregated service systems and live outside spaces where they frequently congregate and socialize with more GBMSM or access LGBTQ+-welcoming services. Thus, youth who are the most vulnerable have less access to HIV prevention services. Implementing tailored interventions and support, either in-person or via online resources, during early adolescence can assist in overcoming systemic factors that can reduce HIV burden in prevention deserts for youth in uniquely vulnerable spaces that limit resilience, psychological well-being, and overall health status.

This study is not without limitations. Analysis at the county level may limit the generalizability of findings to other units of analysis (e.g., individual). Results may also not be generalizable to geographic areas with different HIV burdens, sociodemographics, or areas where local conditions such as polity, policy, and sociocultural norms differ extensively from 
the analyzed regions. In these analyses, we defined service/ provider availability and access using a county level per capita approach, which limits inference. Generally, the county- and regional-level characteristics and the study's results do not account for spatial distance/proximity, in effect assuming that individuals do not cross county lines to access services and that any given service/provider in a county is equally accessible to all residents. However, county-level metrics are important from a public policy standpoint, as individual state's resource allocations in the US are often made at the county level. Similarly, we cannot assert that county-level access reflects individual access to services and providers, particularly for adolescent populations, who may be dependent on parents/ guardians for transportation to providers, especially in rural areas without public transportation options. Additionally, the data presented here represents a 2018 snapshot of access; as recognition of LGBTQ+ healthcare needs and disparities continue to grow, local and national/online provider availability is likely changing. Regular, timely updates of this study will be useful in gauging current resource LGBTQ+-inclusive resource availability, particularly in the post-coronavirus pandemic period, given the disproportionate impact of the pandemic on resources for sexual and gender minorities in the US (Chatterjee et al., 2020).

This study offers an extensive comparative analysis of LGBTQ+ youth-welcoming and affirming resources across different regions of the US and among counties within those regions. We found significant evidence that AGBMSM in many areas in the US do not have equitable access to the resources they may need to effectively navigate the critical transition period from adolescence to adulthood. Multiple counties did not house any providers offering LGBTQ+-inclusive health and wellness programs and resources, highlighting the lived reality of structural vulnerability for many sexual minority youth populations in the US. Access to LGBTQ+ youth-inclusive and affirming resources improves mental and physical health outcomes in the short and long term. Research has shown that having accessible services and resources for youth can lead to better informed and independent decision-making (Beyth-Marom \& Fischoff., 1997; Byrnes, 2002), which can impact better health outcomes over the life course (Viner et al., 2012). Moreover, establishing positive sexual health behaviors in adolescence (e.g., regular HIV and STI testing) can result in better outcomes and habits later in life. Fostering self-efficacy and resilience empowers youth to establish control of their individual health and wellness during a critical period in the life course. Thus, better access to LGBTQ+-inclusive and affirming health and wellness resources could better prepare youth as they enter adulthood. Addressing low service availability may reduce this vulnerable population's disproportionate HIV burden and improve physical and mental health outcomes. Future research may expand upon this descriptive research by investigating geospatial and proximal access to relevant resources in areas with high HIV burdens. Furthermore, geographical analysis may identify trends between location characteristics, underlying demographics, and service provision. Likewise, policy analysis could provide further insight into the sociopolitical landscapes that contribute to the disparities in the distribution of these resources.

Supplementary Information The online version contains supplementary material available at https://doi.org/10.1007/s13178-021-00660-0.

Funding This study is supported by a U01 grant from the National Institute of Minority Health and Health Disparities (1U01MD011274$01)$. Views expressed in this manuscript do not necessarily represent the views of the funding agency.

\section{Declarations}

Conflict of Interest The authors declare no competing interests.

\section{References}

Aidala, A., Cross, J. E., Stall, R., Harre, D., \& Sumartojo, E. (2005). Housing status and HIV risk behaviors: Implications for prevention and policy. AIDS and Behavior, 9(3), 251-265. https://doi. org/10.1007/s10461-005-9000-7

Andrilla, C. H. A., Patterson, D. G., Garberson, L. A., Coulthard, C., \& Larson, E. H. (2018). Geographic variation in the supply of selected behavioral health providers. American Journal of Preventive Medicine, 54(6), S199-S207.

Arreola, S., Santos, G. M., Beck, J., Sundararaj, M., Wilson, P. A., Hebert, P., et al. (2015). Sexual stigma, criminalization, investment, and access to HIV services among men who have sex with men worldwide. AIDS and Behavior. https://doi.org/10.1007/ s10461-014-0869-x

Arrington-Sanders, R., Hailey-Fair, K., Wirtz, A. L., Morgan, A., Brooks, D., Castillo, M., et al. (2020). Role of structural marginalization, HIV stigma, and mistrust on HIV prevention and treatment among young Black Latinx men who have sex with men and transgender women: Perspectives from youth service providers. AIDS Patient Care and STDs. https://doi.org/10. 1089/apc.2019.0165

Bauermeister, J. A., Pingel, E. S., Jadwin-Cakmak, L., Meanley, S., Alapati, D., Moore, M., et al. (2015). The use of mystery shopping for quality assurance evaluations of HIV/STI testing sites offering services to young gay and bisexual men. AIDS and Behavior. https://doi.org/10.1007/s10461-015-1174-z

Bauermeister, J. A., Connochie, D., Eaton, L., Demers, M., \& Stephenson, R. (2017). Geospatial indicators of space and place: A review of multilevel studies of HIV prevention and care outcomes among young men who have sex with men in the United States. Journal of Sex Research, 54(4-5), 446-464. https://doi.org/10.1080/00224499.2016.1271862

Bauermeister, J., Sullivan, P. S., Gravens, L., Wolfe, J., Countryman, K., Smith-Bankhead, N., Drab, R. A., Sallabank, G., Helms, J. D., Khatibi, K., Filipowicz, R., Horvath, K. J., Bonar, E., Castel, A., Hightow-Weidman, L., Guest, J., \& Stephenson, R. (2018). Reducing HIV vulnerability through a multilevel life skills intervention for adolescent men (The iREACH Project): Protocol for a randomized controlled trial. JMIR Research Protocols, 7(7), e10174. 
Bauermeister, J. A., Tingler, R. C., Demers, M., Connochie, D., Gillard, G., Shaver, J., Chavanduka, T., \& Harper, G. W. (2019). Acceptability and preliminary efficacy of an online HIV prevention intervention for single young men who have sex with men seeking partners online: The myDEx Project. AIDS and Behavior, 23(11), 3064-3077.

Beyth-Marom, R., \& Fischoff, B. (1997). Adolescents' decisions about risks: A cognitive perspective. In Health risks and developmental transitions during adolescence.

Bourgois, P., Holmes, S. M., Sue, K., \& Quesada, J. (2017). Structural vulnerability: Operationalizing the concept to address health disparities in clinical care. Academic Medicine. https:// doi.org/10.1097/ACM.0000000000001294

Byrnes, J. P. (2002). The development of decision-making. Journal of Adolescent Health. https://doi.org/10.1016/S1054-139X(02) 00503-7

Centers for Disease Control and Prevention. (2019). HIV in the United States and dependent areas. Division of HIV/AIDS Prevention, National Center for HIV/AIDS, Viral Hepatitis, STD, and TB Prevention, Centers for Disease Control and Prevention.

Chatterjee, S., Biswas, P., \& Guria, R. T. (2020). LGBTQ care at the time of COVID-19. Diabetes \& Metabolic Syndrome, 14(6), 1757.

Coulter, R. W. S., Egan, J. E., Kinsky, S., Friedman, M. R., Eckstrand, K. L., Frankeberger, J., et al. (2019). Mental health, drug, and violence interventions for sexual/gender minorities: A systematic review. Pediatrics, 144(3).

Davis, M. A., Anthopolos, R., Tootoo, J., Titler, M., Bynum, J. P. W., \& Shipman, S. A. (2018). Supply of healthcare providers in relation to county socioeconomic and health status. Journal of General Internal Medicine, 33(4), 412-414.

Drab, R., Wolfe, J. R., Chavanduka, T. M. D., Bonar, E. E., Guest, J. L., Hightow-Weidman, L., Castel, A. D., Horvath, K., Sullivan, P. S., Stephenson, R., \& Bauermeister, J. (2021). Constructing a web-based health directory for adolescent men who have sex with men: Strategies for development and resource verification. Submitted for publication.

Eisenberg, M. E., Erickson, D. J., Gower, A. L., Kne, L., Watson, R. J., Corliss, H. L., \& Saewyc, E. M. (2019). Supportive community resources are associated with lower risk of substance use among lesbian, gay, bisexual, and questioning adolescents in Minnesota. Journal of Youth and Adolescence, 49(4), 836-848. https://doi. org/10.1007/s 10964-019-01100-4

Eisenberg, M. E., Gower, A. L., Watson, R. J., Porta, C. M., \& Saewyc, E. M. (2020). LGBTQ youth-serving organizations: What do they offer and do they protect against emotional distress? Annals of LGBTQ Public and Population Health, 1(1), 63-79. https://doi. org/10.1891/lgbtq.2019-0008

Esri Inc. (2020). ArcGIS Pro (Version 2.6.3). Esri Inc. https://www. esri.com/en-us/arcgis/products/arcgis-pro/

Farmer, G. W., Blosnich, J. R., Jabson, J. M., \& Matthews, D. D. (2016). Gay acres: Sexual orientation differences in health indicators among rural and nonrural individuals. Journal of Rural Health, 32(3), 321-331. https://doi.org/10.1111/jrh.12161

Fisher, C. B., Fried, A. L., Macapagal, K., \& Mustanski, B. (2018). Patient-provider communication barriers and facilitators to HIV and STI preventive services for adolescent MSM. AIDS and Behavior, 22(10), 3417-3428.

Fulcher, C., \& Kaukinen, C. (2005). Mapping and visualizing the location HIV service providers: An exploratory spatial analysis of Toronto neighborhoods. AIDS Care - Psychological and Socio-Medical Aspects of AIDS/HIV. https://doi.org/10.1080/ 09540120512331314312

Gamarel, K. E., Nelson, K. M., Stephenson, R., Rivera, O. J. S., Chiaramonte, D., \& Miller, R. L. (2018). Anticipated HIV stigma and delays in regular HIV testing behaviors among sexually-active young gay, bisexual, and other men who have sex with men and transgender women. AIDS and Behavior, 22(2), 522-530.

Ganapati, S., Ganapati, N. E., La Rosa, M. D., \& Rojas, P. (2010). Spatial disparity of HIV/AIDS service providers: The case of Miami-Dade County. Journal of HIV/AIDS \& Social Services, 9(2), 169-189. https://doi.org/10.1080/15381501003795543

Hatzenbuehler, M. L. (2017). Advancing research on structural stigma and sexual orientation disparities in mental health among youth. Journal of Clinical Child and Adolescent Psychology. https://doi. org/10.1080/15374416.2016.1247360

Hill, S. V., Westfall, A. O., Coyne-Beasley, T., Simpson, T., \& Elopre, L. (2020). Identifying missed opportunities for human immunodeficiency virus pre-exposure prophylaxis during preventive care and reproductive visits in adolescents in the Deep South. Sexually Transmitted Diseases, 47(2), 88-95.

Jadwin-Cakmak, L., Bauermeister, J. A., Cutler, J. M., Loveluck, J., Sirdenis, T. K., Fessler, K. B., et al. (2020). The health access initiative: A training and technical assistance program to improve health care for sexual and gender minority youth. Journal of Adolescent Health, 67(1), 115-122.

Johnson, A. S., Hall, H. I., Hu, X., Lansky, A., Holtgrave, D. R., \& Mermin, J. (2014). Trends in diagnoses of HIV infection in the United States, 2002-2011. JAMA - Journal of the American Medical Association. https://doi.org/10.1001/jama.2014.8534

Kelley, C. F., Rosenberg, E. S., O'Hara, B. M., Frew, P. M., Sanchez, T., Peterson, J. L., et al. (2012). Measuring population transmission risk for HIV: An alternative metric of exposure risk in men who have sex with men (MSM) in the US. PLoS One, 7(12), e53284. https://doi.org/10.1371/journal.pone.0053284

Konrad, T. R., Ellis, A. R., Thomas, K. C., Holzer, C. E., \& Morrissey, J. P. (2009). County-level estimates of need for mental health professionals in the United States. Psychiatric Services, 60(10), 1307-1314.

Latkin, C. A., German, D., Vlahov, D., \& Galea, S. (2013). Neighborhoods and HIV: A social ecological approach to prevention and care. American Psychologist. https://doi.org/10.1037/a0032704

Lucassen, M. F. G., Stasiak, K., Samra, R., Frampton, C. M. A., \& Merry, S. N. (2017). Sexual minority youth and depressive symptoms or depressive disorder: A systematic review and meta-analysis of population-based studies. Australian and New Zealand Journal of Psychiatry. https://doi.org/10.1177/0004867417713664

Mackenzie, S. (2019). Reframing masculinity: Structural vulnerability and HIV among Black men who have sex with men and women. Culture, Health and Sexuality. https://doi.org/10.1080/13691058. 2018.1459845

Maiorana, A., Kegeles, S. M., Brown, S., Williams, R., \& Arnold, E. A. (2019). Substance use, intimate partner violence, history of incarceration and vulnerability to HIV among young Black men who have sex with men in a Southern US city. Culture, Health and Sexuality. https://doi.org/10.1080/13691058.2019.1688395

Martos, A. J., Wilson, P. A., \& Meyer, I. H. (2017). Lesbian, gay, bisexual, and transgender (LGBT) health services in the United States: Origins, evolution, and contemporary landscape. PLoS One, 12(7). https://doi.org/10.1371/journal.pone.0180544

Matsick, J. L., Wardecker, B. M., \& Oswald, F. (2020). Treat sexual stigma to heal health disparities: Improving sexual minorities health outcomes. Policy Insights from the Behavioral and Brain Sciences, 7(2), 205-213.

McInroy, L. B., McCloskey, R. J., Craig, S. L., \& Eaton, A. D. (2019). LGBTQ+ youths community engagement and resource seeking online versus offline. Journal of Technology in Human Services, 37(4), 315-333.

McKenney, J., Sullivan, P. S., Bowles, K. E., Oraka, E., Sanchez, T. H., \& DiNenno, E. (2018). HIV risk behaviors and utilization of prevention services, urban and rural men who have sex with men in the United States: Results from a National Online Survey. 
AIDS and Behavior, 22(7), 2127-2136. https://doi.org/10.1007/ s10461-017-1912-5

Movement Advancement Project. (2020a). Movement Advancement Project: 2020 Tally Report.

Movement Advancment Project. (2020b). Movement Advancement Project: Snapshot LGBTQ Equality by State.

Mustanski, B., Birkett, M., Kuhns, L. M., Latkin, C. A., \& Muth, S. Q. (2015). The role of geographic and network factors in racial disparities in HIV among young men who have sex with men: An egocentric network study. AIDS and Behavior, 19(6), 1037-1047. https://doi.org/10.1007/s10461-014-0955-0

Pantelic, M., Sprague, L., \& Stangl, A. L. (2019). It's not all in your head: Critical knowledge gaps on internalized HIV stigma and a call for integrating social and structural conceptualizations. BMC Infectious Diseases. https://doi.org/10.1186/ s12879-019-3704-1

Parker, C. M., Garcia, J., Philbin, M. M., Wilson, P. A., Parker, R. G., \& Hirsch, J. S. (2017). Social risk, stigma and space: Key concepts for understanding HIV vulnerability among black men who have sex with men in New York City. Culture, Health and Sexuality. https://doi.org/10.1080/13691058.2016.1216604

Parker, T. (2013). Rural-Urban Continuum Codes. http://www.ers.usda. gov/data-products/rural-urban-continuum-codes/documentation.aspx

Pearce, J., Witten, K., \& Bartie, P. (2006). Neighbourhoods and health: A GIS approach to measuring community resource accessibility. Journal of Epidemiology and Community Health. https://doi.org/ 10.1136/jech.2005.043281

Poteat, V. P., Russell, S. T., \& Dewaele, A. (2019). Sexual health risk behavior disparities among male and female adolescents using identity and behavior indicators of sexual orientation. Archives of Sexual Behavior, 48(4), 1087-1097. https://doi.org/10.1007/ s10508-017-1082-6

Rosentel, K., VandeVusse, A., \& Hill, B. J. (2020). Racial and socioeconomic inequity in the spatial distribution of LGBTQ human services: An exploratory analysis of LGBTQ services in Chicago. Sexuality Research and Social Policy. https://doi.org/10.1007/ s13178-019-0374-0

Schober, P., Boer, C., \& Schwarte, L. A. (2018). Correlation coefficients: Appropriate use and interpretation. Anesthesia \& Analgesia, 126(5), 1763-1768.

Siegler, A. J., Wirtz, S., Weber, S., \& Sullivan, P. S. (2017). Developing a web-based geolocated directory of HIV pre-exposure prophylaxis-providing clinics: The PrEP locator protocol and operating procedures. JMIR Public Health and Surveillance. https://doi.org/ 10.2196/publichealth.7902

Sullivan, P. S., Sanchez, T. H., Zlotorzynska, M., Chandler, C. J., Sineath, R. C., Kahle, E., \& Tregear, S. (2020). National trends in HIV preexposure prophylaxis awareness, willingness and use among United States men who have sex with men recruited online, 2013 through 2017. Journal of the International AIDS Society, 23(3), 13. https:// doi.org/10.1002/jia2.25461

Tempalski, B., Beane, S., Cooper, H. L. F., Friedman, S. R., McKetta, S. C., Ibragimov, U., et al. (2020). Structural determinants of Black MSM HIV testing coverage (2011-2016). AIDS and Behavior. https://doi.org/10.1007/s10461-020-02814-4

Turpin, R. E., Salerno, J. P., Rosario, A. D., \& Boekeloo, B. (2020). Victimization, substance use, depression, and sexual risk in adolescent males who have sex with males: A syndemic latent profile analysis. Archives of Sexual Behavior. https://doi.org/10.1007/ s10508-020-01685-Z

United States Census Bureau. (2017). 2016 Poverty and median household income estimates - counties, states, and national. U.S Census Bureau, Small Area Income and Poverty Estimates (SAIPE) Program.

US Department of Health and Human Services, Health Resources \& Service Administration, B. of H. W. (2021). Projecting health workforce supply and demand. Retrieved 1 Apr 2021 from https://bhw.hrsa.gov/dataresearch/projecting-health-workforce-supply-demand

Viner, R. M., Ozer, E. M., Denny, S., Marmot, M., Resnick, M., Fatusi, A., \& Currie, C. (2012). Adolescence and the social determinants of health. The Lancet. https://doi.org/10.1016/s0140-6736(12)60149-4

Walls, N. E., Wisneski, H., \& Kane, S. (2013). School climate, individual support, or both? Gay-straight alliances and the mental health of sexual minority youth. School Social Work Journal.

Watson, R. J., Park, M., Taylor, A. B., Fish, J. N., Corliss, H. L., Eisenberg, M. E., \& Saewyc, E. M. (2020). Associations between communitylevel LGBTQ-supportive factors and substance use among sexual minority adolescents. LGBT Health, 7(2), 82-89.

Zimmermann, H., van Bilsen, W., Boyd, A., Davidovich, U., \& on behalf of HIV Transmission Elimination Initiative Amsterdam (H-TEAM). (2021). P192 Never tested for HIV: Directions for targeted testing interventions among men who have sex with men. Sexually Transmitted Infections, 97(Suppl 1), A108-A108. https:// doi.org/10.1136/sextrans-2021-sti.283

Publisher's Note Springer Nature remains neutral with regard to jurisdictional claims in published maps and institutional affiliations. 